\title{
Calculation and demonstration of dynamic behavior of time dependent electromagnetic field distribution in $\mathrm{H}$-plane rectangular waveguide circuit
}

\author{
Takaharu Hiraoka Hsu, Jui-Pang \\ Faculty of Engineering, Kanagawa University, Yokohama Japan
}

\begin{abstract}
Time dependent electromagnetic field distribution in rectangular waveguide circuits are exactly calculated based on mode corresponding equivalent network and exciting condition. The dynamic behavior of field in waveguide circuit is demonstrated by displaying thus calculated field distribution in time sequence on monitor of personal computer with help of visualization software.
\end{abstract}

\section{INTRODUCTION}

In order to know the performance of microwave circuit in detail, it is effective to demonstrate time dependent electromagnetic field distribution under operation in addition to the calculation of frequency characteristics. The behavior of the microwave circuit can be clearly understood by displaying the dynamic electromagnetic field distribution in time sequence, which is possible by calculating reflected/ transmitted waves of dominant/higher order modes in phaser notation based on the equivalent network.

In this paper, the dynamic behaviors of field distribution for various $\mathrm{H}$-plane rectangular waveguide circuit are calculated by mode corresponding equivalent network and demonstrated. Equivalent network for straight waveguide is represented by mode corresponding multi-transmission line. Also, equivalent network for step type discontinuity is represented by multi-port ideal transformer and that for junction type discontinuity by Foster-type equivalent network. Time dependent electromagnetic field distribution excited by sinusoidal and dominant mode incidence $\left(\mathrm{TE}_{10}\right)$ are calculated from $0^{\circ}$ to $360^{\circ}$ by 1 degree step. Using field visualization software, field distribution in time sequence for various $\mathrm{H}$-plane rectangular circuits are demonstrated on monitor of personal computer at the IMS meeting site.

\section{FIEld Description By Planar Circuit Equations}

$\mathrm{H}$-plane rectangular waveguide circuits having step type discontinuities as shown in Fig. 1 and junction type discontinuity as shown in Fig. 2 can be understood as a parallel plate planar circuit of two dimension with short circuited side wall. Assuming sinusoidal $(\omega)$ and dominant mode $\left(\mathrm{TE}_{10}\right)$ excitation, the field has only $E_{\mathrm{z}}$ and $\boldsymbol{H}_{\mathrm{i}}\left(=H_{x}\right.$, $H_{y}$ ) components and can be described by the following planar circuit equations (1) in phaser notation.

$$
\left\{\begin{aligned}
& \operatorname{grad} \dot{V}=-j \omega \mu_{0} d \dot{\boldsymbol{J}} \\
& \operatorname{div} \dot{J}=-j \frac{\omega \varepsilon_{0}}{d} \dot{V}
\end{aligned}\right.
$$

where planar voltage $\dot{V}(x, y)$ and current density $\dot{J}(x, y)$ are defined by electromagnetic field as in eqs.(2).

$$
\dot{V}(x, y)=-\dot{E}_{z}(x, y) \cdot d \quad \dot{J}(x, y)=\dot{\boldsymbol{H}}_{r}(x, y) \times \boldsymbol{k}
$$

where $k$ is unit vector in $z$-direction.

Once the planar voltage and current density distribution in phaser notation are obtained by solving eqs.(1) under given boundary and excitation conditions, then time or phase $(\theta=\omega t)$ dependent fields under operation are calculated by taking real part of eqs.(3) after calculation of frequency characteristics.

$$
\left\{\begin{array}{l}
V(x, y, t)=\operatorname{Re}\left[\dot{V}(x, y) e^{j \omega x}\right]=\operatorname{Re}\left[\dot{V}(x, y) e^{j \theta}\right] \\
J(x, y, t)=\operatorname{Re}\left[\dot{J}(x, y) e^{j \omega x}\right]=\operatorname{Re}\left[\dot{J}(x, y) e^{j \theta}\right]
\end{array}\right.
$$

\section{EQUIVALENT NETWORK}

Equivalent network for H-plane rectangular waveguide discontinuity are given by modal analysis. The equivalent network for step type discontinuity(Fig.1) is represented by multi-port ideal transformer, and that for junction type discontinuity(Fig.2) by Foster-type equivalent network.

\section{A. Equivalent network for step type discontinuity ${ }^{[1]}$}

H-plane rectangular waveguide cavity shown in Fig.1 (a) has 4 step discontinuities and 5 straight waveguides. Equivalent network for straight waveguide is given by mode corresponding multi-transmission line and that for step discontinuity by multi-port ideal transformer, whose network parameters are given by Table 1 . At step discontinuity, port voltage and current column matrices at both side are related by ideal transformer matrix as eqs.(4).

$$
\boldsymbol{i}^{(j)}=n^{(j),(i)} \cdot \boldsymbol{i}^{(i)} \quad \boldsymbol{v}^{(i)}=\left(\boldsymbol{n}^{(j),(i)}\right)^{t} \cdot \boldsymbol{v}^{(i)}
$$

where $n^{(j),(i)} \equiv\left(\begin{array}{c}n_{q}^{(i),(i)} \\ )\end{array}\right) \quad(i=$ narrow $j=$ wide waveguide) The whole equivalent network is given by cascade connection of them as shown in Fig.1 $(b)$, which leads to the vector notation of bold line in Fig.1(c).

\section{B. Equivalent network for junction type discontinuity}

The right-angle bend with slantwise corner cut shown in Fig.2 $(a)$ is an example of junction type discontinuity, which can be divided into planar waveguides and a planar junction $(S)$. Equivalent network for planar waveguide is given by multi-transmission line, and that for planar junction by Foster-type equivalent network. Eigenmode in Table 2 are once calculated, network parameters for Foster-type equivalent network are easily calculated, i.e., resonant frequency is given by eigenvalue and ideal transformer ratio which means the coupling between planar waveguide mode and planar junction mode is given by overlapping integral of waveguide mode and junction mode. The equivalent network for the whole structure are shown in Fig. $2(b)$, and vector notation by bold line in Fig.2(c). 


\section{CAlCulation Of Time-DEPENDENT FIELD}

A. Cavity coupled to input/output with inductive windows

Structure of rectangular waveguide cavity and its equivalent network are shown in Fig.3(a), and its frequency characteristics are calculated and shown in Fig.3(b). Also, time (phase) dependent planar voltage distribution are calculated and shown in Fig. 3(c) with 45 degree phase step at first resonant frequency $\mathbf{A 1}(f=8.911[\mathrm{GHz}])$.

$B$. Rectangular inductive post in rectangular waveguide

Structure of rectangular inductive post in waveguide and its equivalent network are shown in Fig.4(a). Their frequency characteristics and time dependent planar voltage distribution are shown in Fig.4(b) and (c), respectively.

C. Right-angle bend with corner cut of two kinds

Transmission characteristics of the right-angle bend is improved by cutting the edge squarely or slantly as shown in Fig.5(a), whose calculated frequency characteristics are

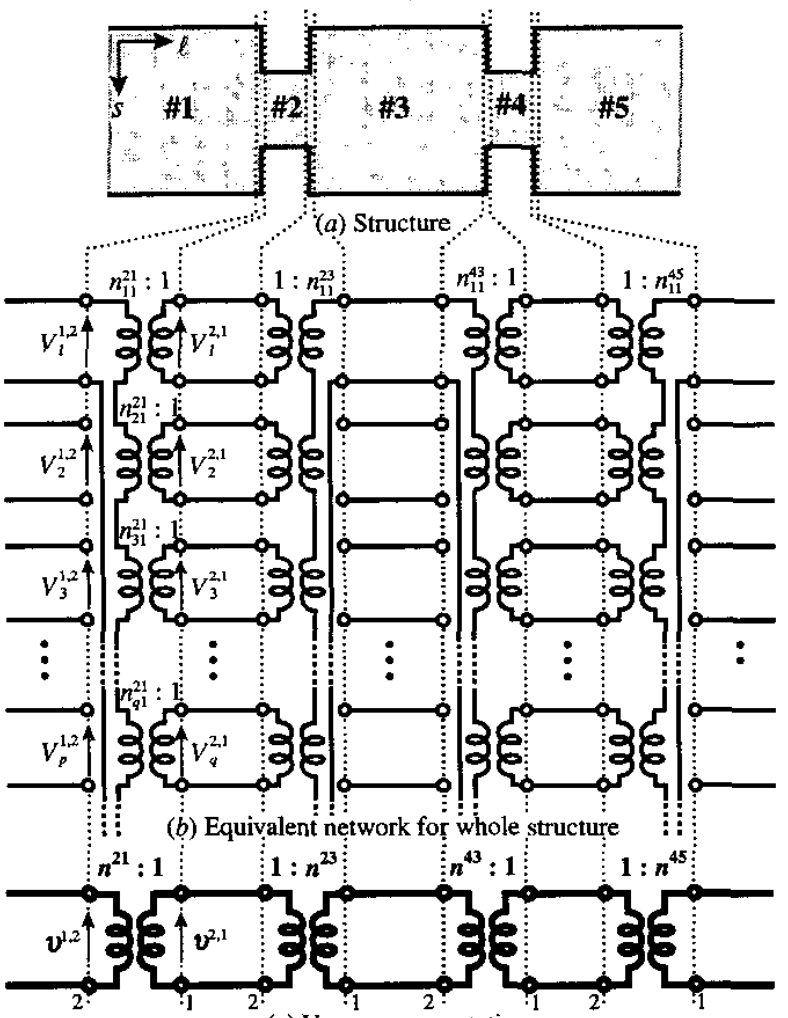

(c) Vector representation

Fig.l Equivalent network for step type discontinuity

$$
\begin{aligned}
& \gamma_{p}^{i}=\sqrt{\left(\frac{p \pi}{W^{i}}\right)-k^{2}} \quad[\mathrm{rad} / \mathrm{m}] \quad Z_{C_{p}}^{i}=\frac{j \omega \mu d}{\gamma_{p}^{i} W^{i}} \quad[\Omega] \\
& \text { The coupling between \#i and \#j waveguide } \\
& \qquad n_{q}^{(j),(i)}=\frac{1}{W^{i}} \int_{0}^{W^{j}} S_{q}^{j}\left(s^{j}\right) S_{p}^{i}\left(s^{i}\right) d s \\
& \text { Eigenmode function along width direction } \\
& \qquad S_{p}^{i}\left(s^{i}\right)=\sqrt{2} \sin \frac{p \pi}{W^{i}} s^{i}
\end{aligned}
$$

Table 1 Network parameter for $i$-th waveguide shown in Fig.5(b) for square cut (A) and slant cut (B), where $\mathrm{C}=c / W$ is cut parameter. Time dependent planar voltage distribution for square and slant cut $\mathrm{C}=0.3$ are shown in Fig.5(c), respectively.

\section{Rectangular waveguide circular right-angle bend ${ }^{123}$}

The structure, its equivalent network and calculated frequency characteristics for various curvature parameter are shown in Fig.6(b), which shows larger the curvature parameter, wider the bandwidth. Time dependent planar voltage for curvature parameter of 0.3 are calculated at normalized frequency $F=1.5$ and shown in Fig.6(c).

\section{CONCLUSION}

An accurate equivalent network method based on mode theory is proposed for waveguide analysis and time-dependent field calculation in microwave circuits. This method is practically applied to various $\mathrm{H}$-plane rectangular waveguide circuit with success.

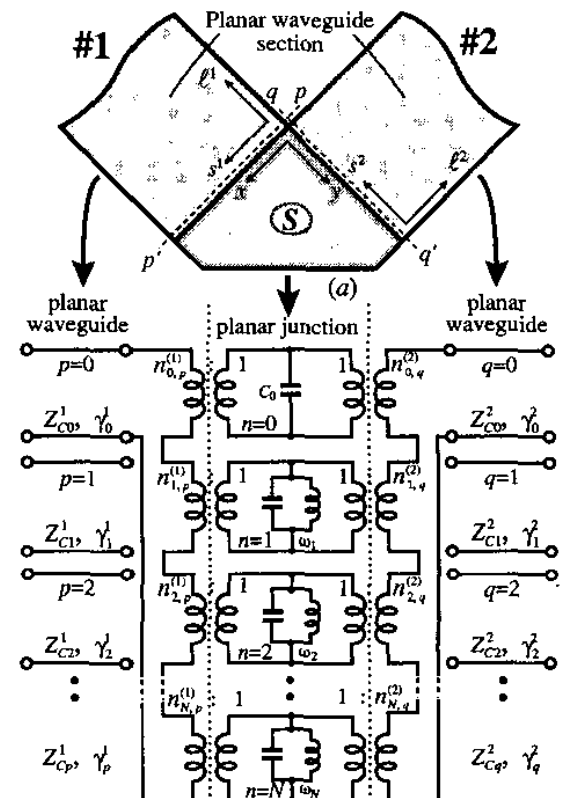

(b) Equivalent network for whole structure

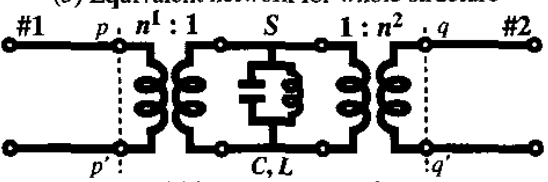

(c) Vector representation

Fig.2 Equivalent network for junction type discontinuity

$\frac{\partial \phi_{n}}{\partial x^{2}}+\frac{\partial \phi_{n}}{\partial y^{2}}+k_{n}^{2} \phi_{n}=0 \quad$ in $S$

$\boldsymbol{n} \cdot \operatorname{grad} \phi_{n}=0 \quad$ on $C \quad$ (entire circumference of $S$ ) $k_{0}=0, k_{1} \leq k_{2} \leq \cdots \quad n=0,1,2, \cdots$

$\frac{1}{S} \iint_{S} \phi_{n} \cdot \phi_{m} d x d y=\delta_{n m} \quad$ (orthonormal)

where $S$ is the area of the planar circuit

Table 2 Eigenmode function for planar junction 


\section{REFERENCE}

[1] J.P. Hsu, T.Hiraoka, H.Honma "Equivalent network for rectangular-waveguide $\mathrm{H}$-plane step discontinuity - Multi-transmission and multi-port ideal transformer-_-" 2000 IEEE MTT-S Di-

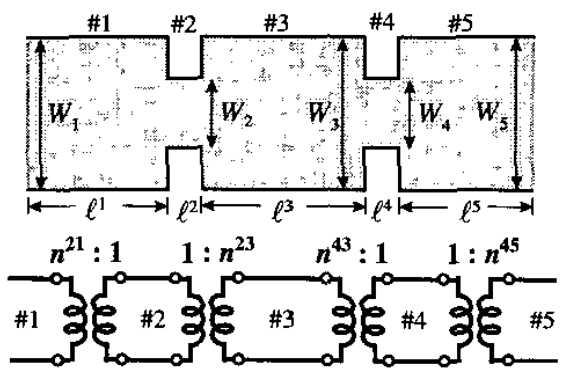

(a) Structure and equivalent network


(b) Frequency characteristics
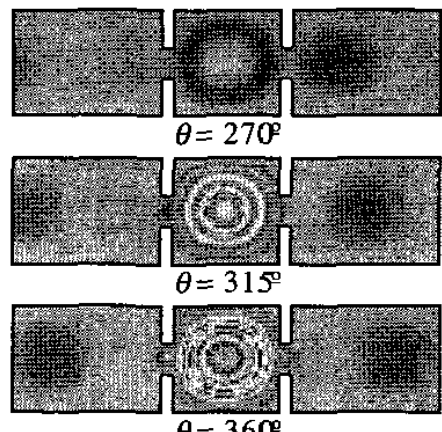

$\theta=360^{\circ}$

Fig. 3 Calculated results for 1 cavity coupled to input/output waveguide with 2 inductive windows

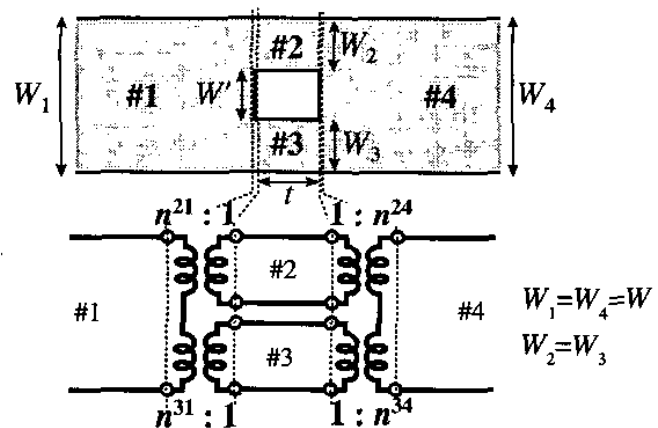

(a) Structure and equivalent network
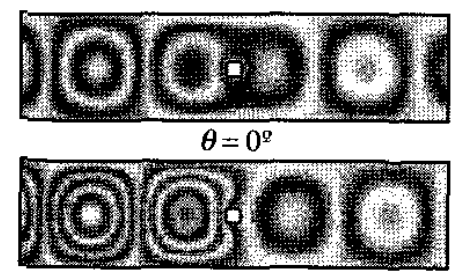

$\theta=45^{\circ}$

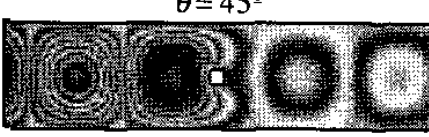

$\theta=90^{\circ}$

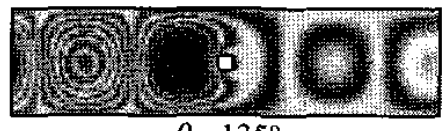

$\theta=135^{\circ}$

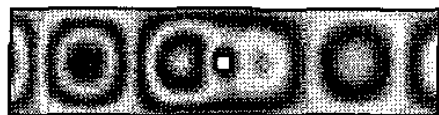

$\theta=180^{\circ}$

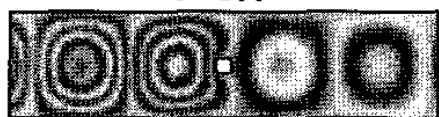

$\theta=225^{\circ}$

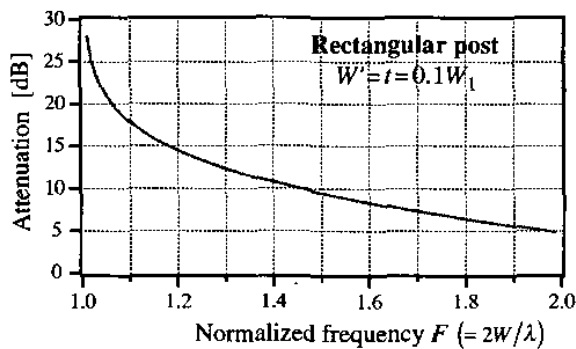

(b) Frequency characteristics

(c) Time (phase) dependent planar voltage distribution at $F=1.5$

Fig.4 Calculated results for rectangular inductive post in rectangular waveguide 


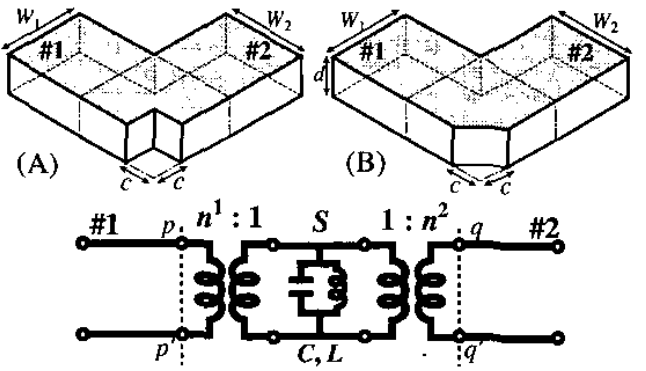

(a) Structure and equivalent network
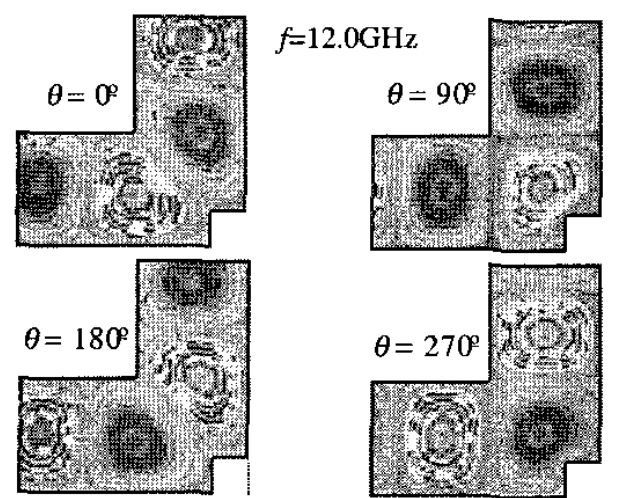

(c) Time (phase) dependent planar voltage distribution of square and slant cut $(\mathrm{C}=0.3)$

Fig.5 Calculated results for $\mathrm{H}$-plane rectangular waveguide right-angle bend with corner cut of two kinds

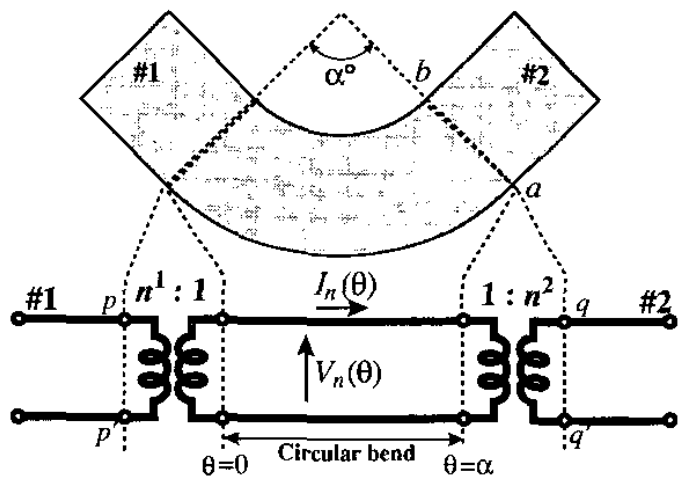

(a) Structure and equivalent network
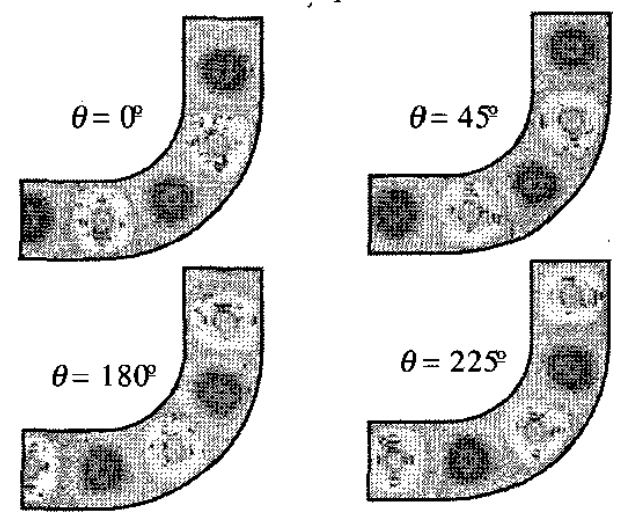

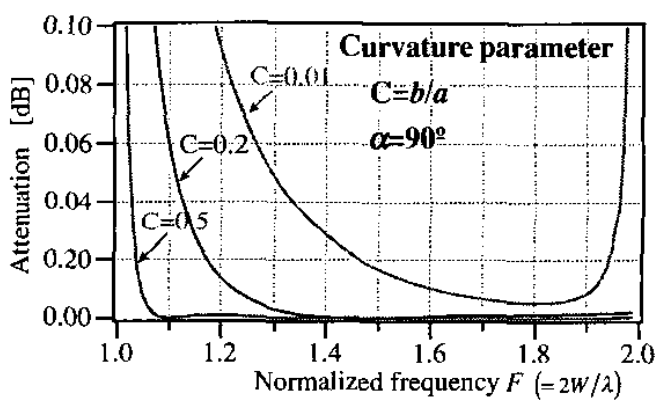

(b) Frequency characteristics

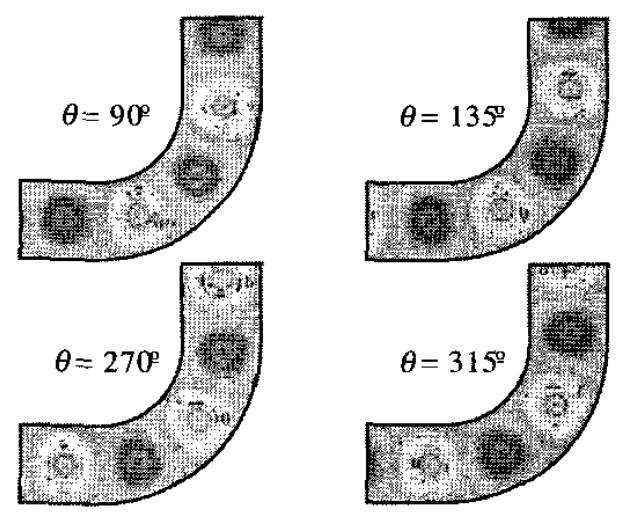

(c) Time (phase) dependent planar voltage distribution for curvature parameter of $\mathrm{C}=0.5$ at $F=1.5$

Fig.6 Calculated results for $\mathrm{H}$-plane rectangular waveguide circular right-angle bend 